\title{
Novas políticas de apoio à pós-graduação: 0 caso FAPEMIG-CAPES
}

- Francisco César Sá Barreto*

- Mario Neto Borges**

\section{Resumo}

Este artigo apresenta um modelo de fomento à pós-graduação adotado em Minas Gerais pela FAPEMIG em parceria com a CAPES. Trata-se de uma experiência inovadora que visa aperfeiçoar e potencializar o uso dos recursos das agências envolvidas ao mesmo tempo em que concentra esforços nas necessidades específicas do Estado. As principais ações deste modelo focam na busca da excelência na pósgraduação de Minas Gerais e na aceleração do processo de formação de doutores e pesquisadores. Os fundamentos para estas ações são baseados na necessidade urgente de acelerar o processo de desenvolvimento científico e tecnológico no país com qualidade compativel com o cenário internacional. Os principais pilares do modelo são descritos em detalhes e podem transformar-se em políticas de fomento de caráter nacional.

Palavras-chave: Pós-graduação. Política de fomento. Qualidade.

\section{New policies for post-graduation courses: the case of FAPEMIG-CAPES Abstract}

This article presents a new model of encouraging graduate courses adopted by FAPEMIG in partnership with the national agency - CAPES, in the state of Minas Gerais. It is an innovative experience which aims to improve and optimize the use of resources while addressing efforts to specific needs of the state. The main actions of this kind of model are focused on achieving excellence in post-graduation courses and are also focused on increasing the speed of PhD's and researchers formation process. These actions are based on the principle that it is urgent to speed up the scientific and technological

\footnotetext{
* Doutor em Física pela University of Pittsburgh; Pós-doutorado, Albert-Ludwigs-Universität Freiburg; Pós-doutorado, Harvard University; Professor Emérito, Universidade Federal de Minas Gerais (UFMG).E-mail: cesar@ufmg.br

** Doutor em Inteligência Artificial Aplicada à Educação, Universidade de Huddersfield, Inglaterra; Professor da Universidade Federal de São João Del Rei. E-mail: marioneto@fapemig.br
} 
development of the country, however, this development must be compatible with the international scenario. The major concepts of the model are described in full and, being successful, they might become a national reference.

Keywords: Post-graduation. Education policies. Education quality.

\section{Nuevas politicas de apoyo al posgrado el caso FAPEMIG-CAPES. Resumen}

Este artículo presenta un modelo para el fomento del post-grado adoptado en el Estado de Minas Gerais por la FAPEMIG en colaboración con la CAPES. Esta es una experiencia innovadora que pretende perfeccionar y potenciar la utilización de los recursos de las agencias que participan y, al mismo tiempo, centrase en las necesidades especificas del Estado. Las principales acciones de este modelo se miran en la búsqueda de la excelencia en postgrado de Minas Gerais y en la aceleración de la formación de doctores y de investigadores. Los fundamentos de estas acciones se basan en la necesidad urgente de acelerar el proceso de desarrollo científico y tecnológico en el país con calidad compatible con el escenario internacional. Los principales pilares del modelo se describen en detalle y pueden convertirse en politicas de fomento de carácter nacional.

Palabras-claves: Post-grado. Política de fomento. Calidad.

\section{Introdução}

O Brasil desenvolveu, nas últimas quatro décadas, um elevado padrão de ciência com uma crescente participação brasileira na produção científica mundial, atingindo $2 \%$ da produção de artigos científicos indexados, ocupando atualmente a $13^{\text {a }}$ posição no ranking mundial. Entretanto, esse desenvolvimento científico ainda não produziu o impacto desejado no setor industrial. A tecnologia e a inovação ainda não se transformaram em atividades rotineiras nos diferentes setores acadêmicos e/ou industriais. Um dos reflexos é a baixa posição do país na produção de patentes e de produtos de base tecnológica. 0 Brasil ocupa hoje a $29^{a}$ posição entre os países no registro de patentes no Escritório Norte-Americano de Patentes e Marcas (USPTO). Essa enorme diferença entre os resultados apresentados pelo Brasil na produção científica e na produção de patentes clama por ações consistentes e urgentes.

0 desenvolvimento industrial brasileiro e a desejável e necessária melhoria da qualidade de nossos processos e produtos, aumentando assim a competitividade no comércio exterior, exigirão ênfases em novos e diferenciados programas nas universidades, na indústria e no governo. É preciso inovar no sen- 
tido definido por Schumpeter (1982), ou seja, um esforço coletivo de transformar ideias, oportunidades e problemas em algo diferenciado. Entre outros aspectos, torna-se necessário a formação de profissionais altamente qualificados e com sua formação em alinhamento com as políticas estratégicas de desenvolvimento estaduais e nacionais.

Políticas consistentes no campo da Ciência, Tecnologia e Inovação (CTCtl), vêm sendo traçadas no nível federal e nos estados. No nível federal os exemplos de mais longo prazo, ou seja na década de 50, são pontuados com as ações da Coordenação de Aperfeiçoamento de Pessoal de Nivel Superior (CAPES) e do Conselho Nacional de Desenvolvimento Científico e Tecnológico (CNPq) na formação de mestres e doutores e apoio às pesquisas científicas. Na década de 60 o exemplo maior é a criação da Fundo de Financiamento de Estudos de Projetos e Programas (FINEP) com vistas ao apoio para o setor empresarial, focando na tecnologia e na inovação. Mais recentemente, pode-se mencionar os Fundos Setoriais que alocam seus recursos no Fundo Nacional de Desenvolvimento Científico e Tecnológico (FNDCT), criado pelo Decreto-Lei $n^{\circ} 719$ (BRASIL, 1969) e restabelecido pela Lei $n^{\circ} 8.172$ (BRASIL, 1991). O FNDCT, atualmente gerido pela FINEP, é a principal fonte de financiamento da CTCtl no âmbito federal. Somem-se ainda iniciativas como a PITCE, o PAC da CTCtl, e a Política de Desenvolvimento Produtivo. No cenário do incentivo à inovação destacam-se, nos últimos anos, a Lei Federal de Inovação, Lei no 10.973 (BRASIL, 2004) e a "Lei do Bem", Lei no 11.196 (BRASIL, 2005).

Nos estados, a partir da Constituição de 1988 (BRASIL, 1998), começa uma nova motivação para a criação das Fundações Estaduais de Amparo à Pesquisa que hoje já somam 23 em todo o País. Estas fundações, nos últimos anos, se organizaram num Conselho Nacional das Fundações de Amparo à Pesquisa (CONFAP). Da mesma forma as Secretarias Estaduais de CTEt se organizaram no Conselho Nacional de Secretários (CONSECTI). Estas articulações estaduais e sua interação com as agências e ministérios no nivel federal levaram, por um lado, a acelerar programas estaduais de apoio a CTCtl inclusive criando condições para a elaboração e sanção das Leis Estaduais de Inovação em vários estados. Por outro lado, levaram a integrar programas, somar recursos e capilarizar ações em todo o País.

Todas estas ações começam a criar um ambiente de legislação e de fomento que pode produzir mudanças importantes para colocar o País em condições de acompanhar a competição mundial. Para isso, é necessário ainda que os programas, atividades e investimentos sejam perenes e - fazer mais e mais rápido. Este trabalho enfatiza uma das novas iniciativas no âmbito estadual em Minas Gerais como resultado da integração das ações da Fundação de Amparo à Pesquisa do Estado de Minas Gerais (FAPEMIG) com a CAPES. 


\section{0 contexto em Minas Gerais}

Em Minas Gerais, a FAPEMIG vive um cenário de significativos investimentos públicos. De fato, como já é de conhecimento público, o governo de Minas Gerais vem repassando desde 2007, à FAPEMIG, os recursos previstos na Constituição Mineira, ou seja, 1\% da receita líquida corrente do Estado. 0 orçamento de 2008 da Fundação atingiu cerca de $R \$$ 230 milhões (FAPEMIG, 2008), para um conjunto de cerca de 6250 pesquisadores doutores de Minas Gerais (Conselho Nacional de Desenvolvimento Científico e Tecnológico (BRASIL, 2006). É importante ressaltar que em 2003 esse mesmo governo, no início de seu primeiro mandato, repassou a essa agência $R$ \$ 23 milhões (FAPEMIG, 2003).

Para avaliar esse montante de recursos, façamos uma comparação com indicadores nacionais e internacionais do investimento por pesquisador. A principal agência estadual de financiamento à pesquisa do país, Fundação de Amparo à Pesquisa de São Paulo (FAPESP), investiu no ano de 2007 o montante de $\mathrm{R} \$ 632$ milhões (FAPESP, 2008). 0 investimento da FAPESP por pesquisador do Estado de São Paulo é de aproximadamente $R$ \$ 32 mil, considerando que esse Estado tem registrado perto de 20.000 pesquisadores doutores em suas instituições de ensino e pesquisa (BRASIL, 2006). 0 Estado de Minas Gerais possui de acordo com os dados do CNPq (BRASIL, 2006) 6250 pesquisadores doutores. Portanto, em 2008, o investimento da FAPEMIG por pesquisador do Estado de Minas Gerais foi de $\mathrm{R} \$ 36$ mil, ou seja, maior que o de São Paulo.

Pode-se também comparar esse investimento da FAPEMIG com os investimentos públicos federais investidos em pesquisa nos EUA. A participação do governo federal americano para a pesquisa representa cerca de $8 \%$ do total investido no pais (NATIONAL SCIENCE FOUNDATION, 2000). Os EUA investiram em 2003 (em moeda constante de 2000) o montante de US\$297.000,00 por pesquisador (RODRIGUEZ; DAHLMAN; SAMI, 2008). Utilizando-se esses dados pode-se inferir que o investimento médio por pesquisador feito pelo governo americano, não incluindo salários, é da ordem de US\$25.000,001. Nessa escala, o pesquisador de Minas Gerais recebe da FAPEMIG o equivalente a $72 \%$ do investimento público federal por pesquisador nos EUA. Os dados utilizados não são do mesmo ano, mas o aspecto que merece destaque é o fato de que os investimentos públicos descritos são da mesma ordem. É importante ressaltar que nessas análises não incluímos os investimentos do Governo Federal através da CAPES, CNPq, FINEP, entre outros. Essas comparações servem para demonstrar que o pesquisador e docente exercendo suas funções no Estado de Minas Gerais recebe apoio da agência estadual recursos comparáveis com de outras agências de governo, no pais e no exterior.

Os investimentos da FAPEMIG nas instituições de ciência, tecnologia e inovação são de várias naturezas. As modalidades de fomento mais importantes e mais utilizadas pela clientela da FAPEMIG são: projetos de pesquisa universal e de demanda

1 Os autores agradecem a Paulo Gazzineli pela obtenção dos dados americanos. 
induzida; apoio a eventos (organização e participação); concessão de bolsas para formação de recursos humanos em ciência e tecnologia; apoio a redes de pesquisa científica e projetos de interesse do Governo do Estado, nas áreas de ciência, tecnologia, inovação e ensino de pós-graduação. Neste trabalho não se pretende descrever os procedimentos de análise e julgamento dos projetos apresentados para receber recursos através dessas diferentes formas de fomento.

Ao lado do fomento direto, a FAPEMIG tem procurado utilizar os recursos estaduais, como contrapartida, para captar mais recursos financeiros, através de parcerias com órgãos federais, iniciativa privada e órgãos internacionais, para o apoio à pesquisa e ao ensino de pós-graduação das instituições de pesquisa e universitárias localizadas no Estado. Para citar apenas alguns exemplos recentes destacam-se as parcerias com o Ministério da Ciência e Tecnologia (MCT), através do CNPq, no caso dos Institutos Nacionais de Ciência e Tecnologia (INCT) e, através da FINEP, no caso do Programa de Apoio à Pesquisa em Empresas (PAPPE), que conta também com recursos do setor empresarial; com o Ministério da Saúde, através do Programa de Pesquisa para o Sistema Único de Saúde (SUS); com o Ministério da Educação, através da CAPES, no apoio à pós-graduação stricto sensu. Apresenta-se, em seguida, com detalhe, a colaboração com a CAPES para apoio à Pós-Graduação em Minas Gerais.

\section{0 cenário da pós-graduação no Brasil}

A pós-graduação é certamente o setor educacional brasileiro de melhor desempenho e que teve ao longo de décadas o planejamento de médio e longo prazos e financiamentos consistentes do Estado Brasileiro. Conforme afirma Cláudio Moura Castro (2002, p. 138),

Sem dúvidas, a PG é a maior realização da educação brasileira em toda a sua história. Conseguimos produzir mestrados e doutorados de padrão internacional, em um país ainda cheio de analfabetos. Lá pelos anos setenta, passamos a Argentina em produção científica, apesar dos seus três prêmios Nobel. E não paramos de aumentar a distância, medida pelo número de publicações incluidas nos periódicos do Current Contents.

0 desempenho da pós-graduação sempre contou com a permanente participação da comunidade acadêmica nacional e foi integrado por ações específicas com a comunidade científica internacional. Além disso, a pós-graduação, desde cedo, incorporou um adequado sistema de avaliação institucional, realizado pela própria comunidade científica das áreas respectivas.

Entretanto, como destacou o Plano Nacional de Pós-Graduação 2005/2010 (BRASIL, 2005), o Sistema Nacional de Pós-Graduação apresenta enormes assimetrias 
em seu funcionamento, tanto do ponto de vista regional, intra-regional e entre estados, como também na evolução de áreas disciplinares tradicionais e de novas áreas na fronteira do conhecimento.

0 diagnóstico dessa situação aponta para a necessidade da formulação de estratégias específicas visando à criação de novos paradigmas para a evolução do sistema, caso contrário, nos próximos anos, se observará à continuidade do crescimento da pós-graduação com a permanência das assimetrias regionais.

Se por um lado não se pode pensar em reduzir os investimentos nos grupos mais qualificados, por outro, torna-se necessário criar condições adequadas para o desenvolvimento dos grupos já estabelecidos em regiões com menor densidade de grupos de pesquisa ou em áreas do conhecimento estratégicas para o desenvolvimento harmônico da ciência e tecnologia nacional. Isso implica estabelecimento de propostas indutoras que contemplem recursos novos e/ou remanejamento de orçamentos. As iniciativas para correção da tendência deveriam começar pelo reconhecimento, por parte dos governos estaduais, da importância da qualificação de recursos humanos locais para propiciar o desenvolvimento do estado e da região.

Para resolver essas assimetrias o PNPG 2005/2010 (BRASIL, 2005) propõe o estabelecimento de programas estratégicos específicos, que serão idealizados e propostos pelas agências, a partir de consultas às universidades, aos institutos de pesquisa, aos órgãos de governo estadual, ao setor empresarial e a outros setores diretamente ligados ao desenvolvimento nacional, que objetivem solucionar cada tipo de assimetria observada. A proposta tem como base uma forte articulação entre as agências de fomento federais (CAPES, CNPq, FINEP) e destas com as Fundações de Amparo e Secretarias de Ciência e Tecnologia dos governos estaduais e com o setor empresarial.

Antes de prosseguir no tema da cooperação entre Agências de Fomento é preciso assinalar que a pós-graduação não esta isolada e não tem vida própria. E por esse motivo seu crescimento depende de vários fatores. De fato, o crescimento da Pós-Graduação está diretamente ligado aos demais níveis de ensino. Ao analisar as matrículas em todos os níveis de ensino, identifica-se um quadro não muito alentador, mas que pode ser usado como um alerta. A pós-graduação só crescerá, se ações imediatas forem adotadas em todos os níveis de ensino. $E_{1}$ além disso, é preciso contar com um importante aliado: o tempo.

Em 2005, estavam matriculados: no ensino fundamental 33.534 .704 alunos; no ensino médio 9.294.579; na graduação 4.453.156; no mestrado 73.980; e, no doutorado 43.958. Nesse mesmo ano, foram titulados 9.874 doutores em todas as áreas. Em Engenharia, obtiveram a graduação 41.556 alunos e foram titulados 1.212 doutores. Esses dados permitem tirar algumas conclusões: 
- De 3.350 alunos matriculados no Ensino Fundamental apenas 1 obtém o Doutorado.

- De 450 alunos matriculados na Graduação apenas 1 obtém o Doutorado.

- De 800 alunos matriculados no Ensino Fundamental 1 obtém a Graduação em Engenharia

- De 30.000 alunos matriculados no Ensino Fundamental 1 obtém o Doutorado em Engenharia.

Ou seja, em relação aos alunos matriculados no ensino fundamental apenas 0,13\% obtém o título de doutor e 0,02\% obtém o doutorado em Engenharia.

\section{0 acordo entre a CAPES e a FAPEMIG para a pós-graduação}

A CAPES apóia diretamente os cursos de pós-graduação do País, e, portanto apóia os cursos localizados em Minas Gerais. Entretanto, acordos de cooperação entre agências podem ser criados, visando atender objetivos específicos e estratégicos e otimizar o uso de recursos. A FAPEMIG tem atuado em consonância com as diretrizes apontadas acima, como descrito em seguida.

No mês de outubro de 2008, a FAPEMIG, motivada pela Secretária Estadual de Ciência Tecnologia e Ensino Superior (SECTES)², assinou com a CAPES um Acordo de Cooperação Técnica e Acadêmica, com recursos financeiros das duas agências, totalizando $\mathrm{R} \$ 10.000 .000,00$, que tem por objeto: (1) apoio aos cursos de pósgraduação localizados no Estado de Minas Gerais avaliados pela CAPES com conceitos 5,6 e 7 ; (2) apoio à capacitação docente das Instituições Públicas e Confessionais de Minas Gerais; (3) apoio na aquisição de equipamentos para cursos de pósgraduação localizados no Estado de Minas Gerais.

Trata-se de uma colaboração - com ênfase na pós-graduação - entre uma agência federal e uma estadual, em moldes inovadores, na medida em que somam esforços e recursos com foco em ações que buscam a excelência nos cursos de pós-graduação por elas apoiados. Por outro lado, o acordo busca acelerar a formação de doutores e pesquisadores no Estado, para dar ao País condições necessárias para não ficar fora da competição internacional de alta tecnologia. Some-se a isso o fato de que a colaboração favorece investimentos centrados em especificidades do Estado que dificilmente seriam atendidas por programas de caráter nacional. Descrevem-se, a seguir, os programas (1) e (2) do Acordo de Cooperação, que induziram novas ações da FAPEMIG, algumas inovadoras.

2 Os autores reconhecem a contribuição do Prof. Evaldo Vilela, Secretário Adjunto, no processo. 


\section{Apoio aos cursos avaliados com conceitos 5, 6 e 7 da CAPES}

A implementação desse programa foi realizada por uma comissão que adotou os critérios que serão descritos em seguida. 0 programa induziu a criação de três novas ações de fomento aos cursos de pós-graduação:

- bolsa conhecimento novo;

- inserção internacional/estágio sanduíche e

- bolsa acoplada.

0 programa e essas novas ações de fomento foram aprovados pelo Conselho Curador da FAPEMIG, que confirmou os critérios utilizados pela referida comissão e indicou a introdução de procedimentos internos para abertura de análises de novos projetos, que poderão ocorrer anualmente.

Existem em Minas Gerais 67 cursos de pós-graduação avaliados pela CAPES com conceitos 5, 6 e 7 (CAPES, 2008). São 44 cursos com conceito 5, 17 cursos com conceito 6 e 6 cursos com conceito 7. Desse conjunto foram analisados 30 cursos, que atendem os requisitos do Plano Mineiro de Desenvolvimento Integrado (PMDI) (MINAS GERAIS, 2000), ou seja, cursos diretamente voltados para a formação de pesquisadores necessários para atender as áreas estratégicas do Estado. Esse foi um requisito básico, definido pela Secretaria de Estado de Ciência, Tecnologia e Ensino Superior, como entidade governamental diretamente interessada nesta ação.

Foram analisadas: as Fichas de Avaliação de cada curso geradas pelas comissões do CTC da CAPES, na última avaliação trienal; a posição relativa de cada curso no conjunto dos cursos classificados com o mesmo conceito; e, no caso dos cursos nível 5, as três últimas avaliações trienais. Nesse último caso o objetivo foi verificar a tendência das avaliações do curso, ou seja, se o conceito crescia, decrescia ou ficava estável. Adotaram-se para a seleção dos cursos que deveriam ser considerados para receber recursos financeiros os seguintes critérios:

- Cursos 7 com tendência a descer para o nível 6;

- Cursos 6 com tendência a serem promovidos para o nível 7;

- Cursos 6 com tendência a descer para o nível 5;

- Cursos 5 com tendência a serem promovidos para o nível 6 .

Foram recomendados para receber apoio cinco cursos com nível 7, cinco programas com possibilidades de promoção do nível 6 para 7 e quatro programas com possibilidades de promoção do nível 5 para 6 .

Os cursos recomendados indicarão qual deve ser o apoio necessário para atingir os objetivos indicados acima. 0 valor de referência do apoio, para cada curso, é de 
$\mathrm{R} \$ 300.000,00$, de uso flexivel. A seleção dos cursos a serem contemplados e a aprovação dos itens de apoio serão de competência de comissões especiais de julgamento por áreas, constituídas para tal fim.

\section{Bolsa: conhecimento novo}

A FAPEMIG criou estímulos aos cursos de nivel 7 para procurarem desenvolver atividades de pesquisa e pós-graduação em temas novos e que representem grandes desafios para as áreas de conhecimento, para os próprios programas de pósgraduação de Minas Gerais, e que, portanto, tenham possibilidades, se bem sucedidos, de causar impacto na ciência brasileira e mundial.

A proposta é apoiar os cursos de nivel 7, por meio de investimento, na forma de uma bolsa e custeio, denominada Bolsa Conhecimento Novo, a ser oferecida por um período de até 3 anos a pesquisador talentoso, diferenciado em relação ao conjunto normal, que proponha e tenha liberdade de realizar pesquisas em temas não explorados, na fronteira do conhecimento, e que carreguem um grande risco de "incerteza". Abordar o tema desafiante, com perspectivas de criação do "Novo" é, em si, um dos objetivos da Bolsa. Os cursos de nível 7 são convidados a apresentar os temas a serem pesquisados. As propostas são analisadas por consultores externos, do Brasil e do Exterior. As propostas aprovadas são anunciadas para contratação dos bolsistas. 0 apoio global será de $\mathrm{R} \$ 300.000,00$ por três anos, compreendendo as parcelas da bolsa e o restante para custeio, a ser usado de forma flexível.

\section{Inserção internacional: estágio sanduíche}

Outro novo programa visa a um atendimento diferenciado de intercâmbio internacional aos cursos de pós-graduação. Os cursos de nível 6 e 7 são considerados de padrão internacional e, portanto, deveriam ter como parte essencial na formação de doutores, estágios de curta duração em instituições internacionais de excelência. Para atender a essa proposta, as bolsas concedidas pela FAPEMIG a alunos de doutorado de cursos de nível 6 e 7, terão, a partir de 2009, como requisito, entre os vários existentes, a realização de um estágio sanduíche em instituição estrangeira de alto nivel acadêmico. Os recursos para essa atividade fazem parte do apoio concedido pela FAPEMIG. Essa mobilidade estudantil trará enormes benefícios aos cursos, a Minas Gerais e ao País. Como exemplos: experiência do aluno em instituição estrangeira, proficiência em língua estrangeira, possibilidade de criação de vínculos de pesquisa entre grupos nacionais e estrangeiros, maior difusão dos grupos nacionais de pesquisa em outras instituições estrangeiras.

\section{Bolsa acoplada}

0 Estado de Minas Gerais por meio de bolsas de pós-graduação atende estudantes oriundos de cursos de graduação de instituições localizadas no Estado e de fora do Estado. Portanto, incentivos para permitir a permanência no Estado de recém-doutores formados em programas de pós-graduação de instituições mineiras são importantes. 
Dessa forma, procurando atender a essa política de fixação de doutores no Estado, a FAPEMIG cria em 2009 um novo programa para estudantes de doutorado das universidades mineiras. Os estudantes que desejarem poderão se candidatar ao programa Bolsa Acoplada, constituído de um estágio sanduiche no exterior, nos moldes descritos no subitem 3.1.2 acima, seguido de uma bolsa de pós-doutorado em instituição de Minas Gerais, de duração máxima de três anos.

São vários os resultados esperados desse tipo de programa. 0 estudante contemplado com essa bolsa adquiriria uma experiência no exterior, ainda no período de formação - período do sanduíche no qual criaria vínculos de pesquisa com a instituição do exterior, e, em seguida à obtenção do doutorado, desenvolveria suas atividades de pesquisa e ensino em instituição mineira, antes de obter uma posição de emprego permanente em alguma instituição de ensino ou pesquisa ou no setor empresarial.

\section{Programa Mineiro de Capacitação Docente (PMCD)}

0 Estado de Minas Gerais conta com quatorze Instituições Públicas de Ensino Superior, sendo doze federais e duas estaduais, além de uma Instituição Confessional, considerada de interesse público. 0 quadro docente dessas instituições apresenta uma realidade que precisa ser modificada. Trata-se da titulação dos docentes. Do conjunto de docentes dessas quinze instituições, na média, 39\% possuem o doutorado, 31\% possuem o mestrado e 29\% não possuem titulação pós-graduada. Diante dessa situação, a FAPEMIG criou 0 Programa Mineiro de Capacitação Docente (PMCD), com o objetivo de aumentar em poucos anos a titulação no nível de doutorado dos docentes dessas instituições mineiras.

0 programa visa a promover a melhoria das atividades de ensino e pesquisa dessas Instituições de Ensino Superior (IES) apoiando os esforços institucionais de capacitação e aprimoramento da qualificação de seus docentes. 0 Programa pressupõe o desenvolvimento de projetos de cooperação entre as IES de Minas Gerais acima mencionadas com o seguinte credenciamento:

- Instituições de Origem - são aquelas que necessariamente devem demonstrar o esforço institucional em aprimorar suas atividades de ensino, pesquisa e pós-graduação apoiando a titulação dos seus docentes (que ainda não têm doutorado) e

- Instituições de Destino - são aquelas que oferecem vagas extras em seus cursos de pós-graduação com nível 5 ou maior para as demais IES de origem.

As IES credenciadas como Destino, ou seja, as que oferecerão as vagas para os candidatos ao doutorado, devem possuir pelo menos um curso de pós-graduação com conceito igual ou superior a 5, na avaliação CAPES referente ao triênio 20042006. As IES credenciadas como Origem, ou seja, aquelas que vão enviar seus do- 
centes para obtenção do doutorado, devem apresentar à FAPEMIG o plano institucional de qualificação de seus docentes, incluindo necessariamente perspectivas de trabalho dos docentes participantes após a titulação.

Atendendo o convite formulado pela FAPEMIG foi encaminhada pelas IES de Origem uma demanda total de 544 candidatos ao Doutorado. Uma comissão formada de pró-reitores de pós-graduação de universidades de Minas Gerais analisou a demanda e disponibilidade de recursos, e propôs que fossem distribuídas 231 vagas à estas IES de Origem. A FAPEMIG enviou ofício às mesmas solicitando a indicação de candidatos, em ordem de prioridade, indicando a área de titulação e a instituição de titulação pretendidas. A seleção dos candidatos foi feita pelos cursos das IES de Destino. Os candidatos aceitos pelos programas de pós-graduação das IES de Destino recebem bolsa total ou parcial para realização do doutorado. A bolsa total é concedida por 18 meses aos candidatos que cursarem o doutorado em Instituição de Destino fora de seu domicílio. A bolsa parcial é concedida, pelo mesmo período, àqueles que cursarem o doutorado na mesma cidade que residem. As bolsas poderão ser concedidas por mais 6 meses por ocasião da elaboração final da tese - caso ela seja desenvolvida em Instituição de Destino fora da cidade de residência do candidato. É importante ressaltar que essas bolsas não fazem parte do conjunto de bolsas distribuídas, tanto pela CAPES como pela FAPEMIG, anualmente aos programas de pós-graduação. Isto é, são bolsas adicionais àquelas já existentes nos programas convencionais.

O PMCD, inclui ainda em sua concepção, a concessão de recursos de custeio denominada Taxa de Bancada para as Instituições de Destino. As Taxas de Bancada se destinam a serem aplicadas nos projetos de doutoramento dos candidatos do Programa. Dessa forma as Instituições de Destino recebem um apoio adicional pelo fato de estarem oferecendo vagas extras àquelas previstas anualmente em seus cursos de pós-graduação. 0 número de Taxas de Bancada oferecidas é igual ao número de candidatos selecionados e são concedidas também em 18 mensalidades podendo chegar a 24 caso os candidatos venham a elaborar suas teses nessas Instituições de Destino. O PMCD já está em vigência desde maio de 2009.

\section{Considerações finais}

É desejável que o fluxo de recursos públicos de Minas Gerais continue acompanhando o crescimento do corpo de pesquisadores e as demandas do País, em geral, e do Estado de Minas Gerais, em particular. Os próximos governos de Minas Gerais devem tratar esse financiamento na perspectiva maior de política de estado e não, simplesmente, de política de governo. Esse tipo de apoio público é estratégico para o futuro do País e do Estado.

Por outro lado, espera-se que o setor privado, através das empresas instaladas no Estado, acompanhe essa iniciativa do governo estadual e inicie uma política de 
apoio à pesquisa, tecnologia e inovação compatível com o investimento público ora realizado. Esse apoio do setor privado pode ser feito diretamente nas empresas, nos seus centros de pesquisa, desenvolvimento e inovação, através de projetos em parceria com grupos de pesquisa de universidades localizadas no Estado, ou ainda em parceria com a FAPEMIG.

É também esperado que a comunidade de pesquisa use esse investimento para promover o crescimento do corpo de pesquisadores e da qualidade da pesquisa gerada nas instituições. Ou seja, que as demandas da comunidade cresçam em número, para garantir uma seleção mais competitiva e que apresentem as qualidades exigidas para seu financiamento em padrões de excelência competitiva em nível nacional e internacional.

Finalmente, espera-se que os recursos aplicados pela FAPEMIG, ao lado de outros esforços, das universidades, setor privado e governo federal, ajudem a criação de condições para o aparecimento de resultados mais eficazes de desenvolvimento. Desenvolvimento este que apresente perspectivas de médio e longo prazo para promover um salto qualitativo e quantitativo da ciência, da tecnologia e da inovação no Estado e no País.

\section{Referências}

BRASIL. Decreto-Lei no. 719, de 31 de julho de 1969. Cria o Fundo Nacional de Desenvolvimento Científico e Tecnológico e dá outras providências. Diário Oficial da União, Brasilia, DF, 31 jul. 1969.

. Lei $n^{\circ} .8 .172$, de 18 de janeiro de 1991. Restabelece o Fundo Nacional de

Desenvolvimento Científico e Tecnológico. Diário Oficial da União, Brasilia, DF, 22 jan. 1991. Disponivel em: <http://www.planalto.gov.br/ccivil/LEIS/L8172.htm>. Acesso em: 23 nov. 2009.

. Lei $n^{\circ} 10.973$, de 2 de dezembro de 2004. Dispõe sobre incentivos à inovação e à pesquisa científica e tecnológica no ambiente produtivo e dá outras providências. Diário Oficial da União, Brasília, DF, 3 dez. 2004.

. Lei no. 11.196, de 21 de novembro de 2005. Institui o Regime Especial de Tributação para a Plataforma de Exportação de Serviços de Tecnologia da Informação. Diário Oficial da União, Brasília, DF, 22 nov. 2005. Disponível em: <http://www.planalto.gov.br/ccivil_03/_Ato2004-2006/2005/LEI/L11196.htm>. Acesso em: 13 nov. 2009.

- Ministério da Educação. Plano Nacional de Pós-Graduação - PNPG 2005-2010. Brasilia, DF, 2005. 
CAPES. Pós-graduação. Brasília, DF, 2008. Disponivel em: <www.capes.gov.br>. Acesso em: 10 nov. 2009.

CASTRO, C. M. A pós-graduação e a CAPES: o que deu certo e o que deve ser feito. Infocapes, v. 10, n. 2, p. 138-143, 2002.

CNPq. Apresentação. Brasilia, DF, 2006. Disponivel em: <www.cnpq.br>. Acesso em: 10 nov. 2009.

FAPEMIG. Belo Horizonte, 2008. Disponivel em: <www.fapemig.br>. Acesso em: 10 nov. 2009.

FAPESP. São Paulo, 2009. Disponivel em:<www.fapesp.br>. Acesso em: 10 nov. 2009.

MINAS GERAIS. Secretaria de Estado de Planejamento e Gestão. Plano Mineiro de Desenvolvimento Integrado. Minas on Line, Belo Horizonte, 2000. Disponivel em: $<$ www.planejamento.mg.gov.br/governo/publicacoes/ plano_mineiro_des_integrado.asp>. Acesso em: 12 nov. 2009.

NATIONAL SCIENCE FOUNDATION. Statistics, Arlington, Virginia, 2000. Disponível em: <www.nsf.gov/statistics>. Acesso em: 13 nov. 2009.

RODRIGUEZ, A.; DAHLMAN, C.; SAMI, J. Conhecimento e inovação para a competitividade. Brasília, DF: Banco Mundial: CNI, 2008.

SCHUMPETER, J. A. A Teoria do Desenvolvimento Econômico. São Paulo: Abril Cultural, 1982.

Recebido em: 10/07/2009

Aceito para publicação em: 04/09/2009 